\title{
A critical assessment of a partially-successful analytical campaign
}

\author{
$\underline{\text { P B Williams }}$ \\ Land Capability Analysis, Defence Science and Technology Group \\ Email: Peter.Williams@dst.defence.gov.au
}

\begin{abstract}
In 2016, the author led a small team undertaking an investigation into capability acquisition and concept development for Army Headquarters. The ensuing analytical campaign spanned a dozen workshops and a week-long Limited Objective Experiment, with significant modelling work done over the course of twelve months. Whilst the final outcomes enabled the sponsor to meet a number of critical decision points, the overall objectives were only partially met and a large amount of work was ultimately fruitless. After a critical self-review of the analysis campaign, a set of distinct points of failure have been identified and, from these, valuable lessons have been extracted to take forth into future analytical campaigns.
\end{abstract}

Keywords: Analytical campaigns, experimentation, lessons learned 


\section{INTRODUCTION}

In 2016, the Land Capability Analysis (LCA) Branch of the Defence Science and Technology Group led an analytical campaign (The Technical Cooperation Program, 2006) to assist Special Operations Command (SOCOMD) in understanding the requirements of a dedicated Special Recovery Operations (SRO) capability. Whilst successful in delivering the requisite findings to the client, a number of clear failings in the planning and execution of the campaign were observed. These flaws wasted precious resources and ultimately resulted in poorer quality outputs.

This paper seeks to highlight the planning aspects that required improvement and then identify lessons than can be learned in the wake of the study.

\subsection{Land Capability Analysis and Experimentation Research Management}

The primary focus of the LCA work program is to address the needs of Army Headquarters (AHQ) in understanding the capabilities, concepts and structures required for Australian land forces in the mid-term future. This effort seeks to set decision points and milestones for how the Army should posture itself in approximately 25 years into the future, so that decisions and projects can be implemented in the near future to meet them.

Working in intimate collaboration with LCA is the Experimentation Research Management ${ }^{1}$ (ERM) cell within AHQ. This group comprises a number of military staff who support Army Modernisation by being close conduits into AHQ and Defence, providing domain expertise and guidance to analysts, and in assisting in the execution of activities.

Together, LCA and ERM solve a set of studies each year that guide the evolution of Army capabilities, concepts and structures.

\section{THE SPECIAL RECOVERY OPERATIONS ANALYTICAL CAMPAIGN}

One of the studies assigned to the LCA team and undertaken in 2016 was aimed at addressing the use of funds identified in the 2015 Defence White Paper (Department of Defence, 2015) for the expansion of the dedicated Special Operations (SO) Rotary Wing (RW) fleet; specifically for use in the SRO mission set.

\subsection{Special Recovery Operations}

Special Recovery Operations are a SOCOMD mission set and, as defined by Davies et. al. (2014), are undertaken to rescue personnel or seize equipment from permissive, uncertain or hostile environments. They include domestic and offshore counterterrorism (CT) operations and non-combatant evacuation operations, as well as personnel recovery.

There are a variety of means by which a Tactical Assault Group ${ }^{2}$ might approach the objective within an SRO mission. These include: on foot; by land vehicles (such as Humvees or quad bikes); by parachute; by boat; or by helicopter. To meet one of the aims of the client, this study was restricted to only investigating the helicopter approach option.

Nested within a broad topic statement were the six Analytical Questions to be answered:

1. What are the gaps in the SRO capability and what is the impact of these gaps?

2. What options could address the need for rapidly deployable RW and Intelligence, Surveillance and Reconnaissance?

3. What are the Australian Defence Force's needs to project SRO globally?

4. What options could address the remaining issues which limit global SRO?

5. Which options meet the needs of global SRO but provide the greatest flexibility to the wider Army?

6. What are the special versus conventional training implications?

\subsection{Additional Support}

Additional support was supplied to the analytical team over the course of the campaign in the form of a Special Operations (SO) subject matter expert (SME) from AHQ. He filled a vital role in providing detailed

\footnotetext{
${ }^{1}$ Currently known as Land Warfare Lab

${ }^{2}$ A Tactical Assault Group (or TAG) is a unit within the Special Forces whose specific skills and purpose is to conduct domestic Counter-Terrorism and global Special Recovery Operations.
} 
domain knowledge, serving as an invaluable link to various data sources, guiding the analytical plan and injecting the required enthusiasm into SOCOMD to ensure the relevant participants attended a series of activities.

\subsection{Timeline}

Figure 1 shows the timeline for the analytical campaign. After the initial problem scoping phase, a chain of one- and two-day workshops were used to generate data and understanding, which culminated in a week-long Limited Objective Experiment (LOE) in October 2016. The data from all of the activities was then analysed and findings reported to AHQ and SOCOMD in 2017.

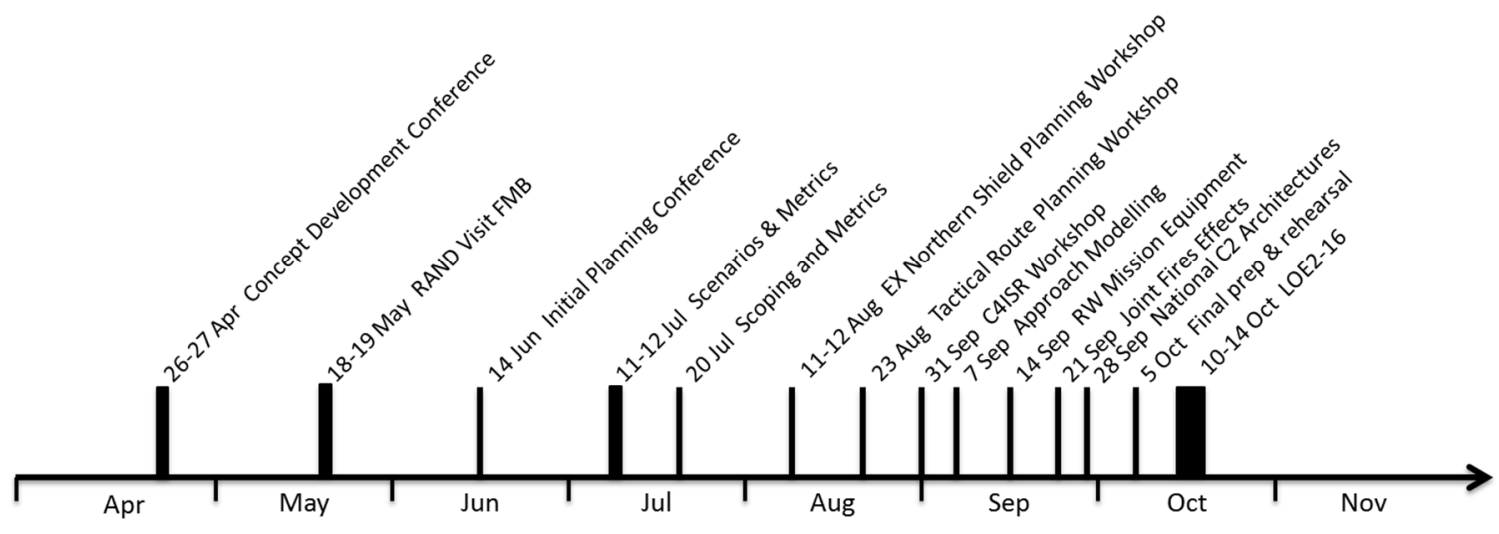

Figure 1. Timeline for the Analytical Campaign

\section{ASSESSMENT OF THE ANALYTICAL CAMPAIGN}

The six analytical questions are summarised in Figure 2, along with a crude assessment, based on the author's professional judgement, of how successfully they were addressed in the final analysis.

\section{What are the gaps, and the impacts of gaps? \\ What are the optimal RW options? \\ What are the ADF's needs to project globally? \\ What are the remaining issues? \\ Which options provide flexibility to Army? Special vs conventional training implicatons?}

Figure 2. Assessments for Analytical Questions

It was assessed that, on the whole, only about $30 \%$ of the entire problem space was resolved to any notable level of rigour. Whilst this was sufficient for the sponsor to argue for the key needs of the project, it meant that the study was only partially successful in achieving its overall objectives.

\section{SOURCES OF FAILURE}

Following a critical review of the work program for the year, a range of distinct sources of localised failure were identified. These are described below in detail, in no particular order of importance.

\subsection{Scope of the problem space}

The six analytical questions which underpinned the study were extremely disparate in scale and focus. Some aspects were technical (such as comparisons of on-board sensors and communications systems); some were tactical (such as the ability of light, medium or heavy helicopters to hover or land on a given target); some were operational (such as the ability to project different fleet sizes and types into theatre); and some were life-of-type issues (the implications on training for all SRO support personnel). 
These variations made it difficult to design an analytical campaign to model and investigate them all within the available timeline and resources. Additionally, each aspect required a different modelling technique and different expertise. This was exacerbated by the interdependencies between the different facets of the problem.

\subsection{Chaotic nature of the data}

Not only did every component of the study affect many others, thereby forming a complex system, but tiny variations in any one parameter could have had significant and unpredictable consequences elsewhere, whereby making it a chaotic system (Kaneko and Tsuda, 2011).

By way of example, consider the following scenario: a given helicopter platform is being used to lift ten SO operatives to the target. Through the process of wargaming, it is determined that the platform needs additional armour to increase survivability against small arms fire. The additional weight means that, given a range of distances, temperatures and altitudes, the helicopter can no longer make it to the target without running out of fuel. Therefore, the ten operatives are split over two helicopters. This then means that, with an operational availability of $\sim 50 \%$, four platforms need to be projected into theatre instead of two. Since three can be loaded into a single C-17, now two of these lift platforms are needed to transport the fleet into theatre, within the timelines of an SRO mission. And then they (and the flight crew, and ground crew) must be available in this small window...

From this simple example it can be seen that modelling the SRO 'system' contains a number of sub-models, at every conceivable scale, and even minute changes in any component can have a large impact on any and all other components of the system.

\subsection{Understanding of the analytical questions}

It is common for analytical questions from a client to be worded obtusely and open to interpretation. Indeed, it is one of the roles of the analyst to seek out the true nature of the problem to be solved (Curtis et al, 2005). In this particular instance, the sponsor lay directly above ERM in their command structure, and was a reasonably high-ranking officer who reported directly to a 1-star officer. An artefact of this relationship was that the ERM liaisons behaved in a very 'protective' fashion with respect to disturbing the client and wasting their time. Through the early scoping phases of the study, the analytical team was both discouraged from contacting the client directly and reprimanded when it did so. After several of these episodes, the analytical team relented and relied on the client's subordinates to act as proxies.

Thus, on the occasions when clarity was sought on the underlying intent of the analytical questions, a subjective synthesis was received an intermediary based on interpretation and speculation. Therefore, the analytical plan was designed based on the hope that it could produce outputs to meet the client's needs.

\subsection{Lack of Investigator Triangulation}

Investigator Triangulation (Guion et al., 2011) is based on using a range of analysts with disparate backgrounds, experience and biases to view a problem and collaboratively evolve a solution. Effective Investigator Triangulation is one of the strengths of LCA (Shine and Kempt, 2017).

However, for this study, only a single analyst was involved in scoping the problem and generating methods for solution. Thus, there were no opposing views, additional ideas or internal 'red teaming' of the plan. As such, the campaign suffered, particularly when the workload from the scope and volume of the problem space overloaded the lone investigator.

\subsection{Analysis Tempo vs Execution Cycle}

The timeline presented in Figure 1 was coordinated by a single analyst; the SO liaison officer worked hard to secure participants and facilities, but the development of plans, data capture, data analysis and ensuring the holistic campaign was being delivered rested with just one person. Thus, for several months through the core of the study, the analyst's battle rhythm was similar to that shown in Figure 3 . This is referred to as the 'workshop execution cycle'. 


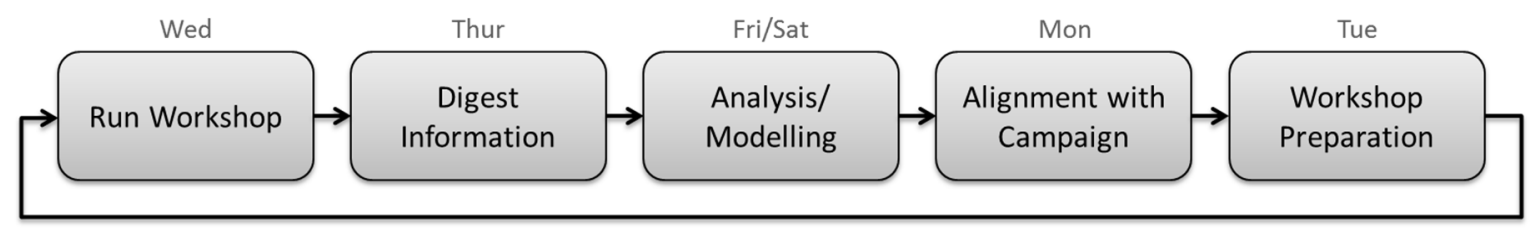

Figure 3. The Workshop Execution Cycle

As topics required more time to analyse, the critical 'alignment with campaign' step would be impinged upon and, in many cases, the 'preparation' phase would be also truncated, thus leading to poorer data collection.

\subsection{SME bias and false positives}

As the analysis tempo began to be overrun by the Execution Cycle, significant concerns were expressed to the SO liaison officer. A response would subsequently be received and assurances given that the sponsor was happy with the progress of the study. It later became clear that these assurances were ill-informed and needed to be addressed.

Following much reflection of the process, one conclusion was that there was a disconnect in communication between the analyst and the stakeholders. From a non-analytical perspective things were in fact progressing well: there were many workshops; many pertinent topics being discussed; many passionate people engaging in conversations and leveraging off years of experience. However, from an analytical perspective, objective, defensible data was required; models of systems were needed that would stand up to scrutiny and contestability. As the analysis was being overwhelmed by the execution, the usable data became more and more sparse. Yet the sponsor was seemingly comfortable with the progress of the activity; this perception should have been challenged.

\subsection{Inexperienced LOE team}

In section Error! Reference source not found. the analytical team was introduced and they had a substantial involvement in delivering the requirements for the activity. The Aerospace Capability Analysis (ACA) team in particular provided an excellent contribution to the analysis. However, in terms of experience in data collection and knowledge elicitation roles at an LOE, none of the ACA team members had been involved in any activities before.

Being able to operate under very limited guidance (little more than an understanding of the problem statement) and then knowing when to inject the right questions to a group of experts engaging in free-flowing discussions is in itself a skill. Most staff in LCA have spent a number of years developing and refining this skill to some extent. However during the LOE, it soon became apparent that the team were trying to build these skills during the activity, having not had the suitable training, preparation or guidance that was required.

\section{LESSONS LEARNED}

It is easy to reflect on a job well done and enjoy success. But it is valuable to reflect on sub-standard performances and learn from those mistakes. As noted by Dörner (Dörner, 1990), "Self-reflexive examination and critique of one's own way of acting is an essential means of adapting one's own way of acting to the given circumstances. Dispensing with self-reflection is therefore a major error."

Many of the findings listed below are self-explanatory; the objective of this paper is not to be new approaches, but to warn practitioners, even with years of experience to remain vigilant.

\section{Lesson 1: Always have 2-3 'leading' analysts}

Every study has a Lead Analyst. And most will have a number of analysts who contribute to (and see) only a small part of the complete study. But every design phase should include at least 2-3 'leading' analysts who have vision of the full scope of the study, and can contribute ideas at all times, even if only to critique other ideas of the team (Zenko, 2015).

\section{Lesson 2: Insist on (and use) a direct line to the sponsor}


The sponsor of the study is likely to be a 1-star or 2-star military officer, and their time is extremely valuable and limited. But the benefits of talking directly to them - and preferably in person - far outweigh the risks of misunderstanding their intent and expectations and failing to deliver useful products months later. It is essential that the analysis team make it clear to them that early and frequent engagement is essential to their cause and a few hours of their time will make a significant positive impact.

\section{Lesson 3: Strong leadership is important}

Strong leadership is critical in a campaign such as this. On reflection of the study, two main areas that suffered from a lack of strong leadership were identified.

Firstly, there was no clear articulation of the analysis plan to the other analysts involved (particularly in the lead up and execution of the LOE). This was due in part to having a poor plan, not managing the data capture during the LOE sufficiently, and unreasonable expectations of the experience of the other analysts.

Secondly, management of workload and expectations of staff was not clearly articulated. At times, analysts delivered less than what was expected. This was accepted by the lead analyst, who proceeded to undertake the completion of the work himself. This was assessed as being an inefficient way of managing workload, in addition to being detrimental to the skill development of the analyst team.

\section{Lesson 4: Better communication is required to address the balance between the Analysis Tempo and the Execution Cycle}

Good, clear communication is a challenge within many organisations, and a lack of communication and clarity has at times caused issues in the experimentation process in the past. LCA has worked with the ERM over a number of years who have organised workshops and experiments for them by identifying the critical participants, booking their time and bringing all the necessary resources together. This can be a difficult task, particularly with most SMEs being extremely busy and unable to commit to anything without sufficient forewarning. Therefore, ERM request that any activities be scheduled months in advance. Issues arise when several weeks are required to prepare for such activities, and a number of aspects need to be completed, including scoping the problem, undertaking initial investigations, commencement of modelling, and understanding the remaining questions that need to be addressed at a workshop.

Thus, if the analysis preparation to be completed before the Execution Cycle commences, it could be many months before valuable outcomes is produced. In order to shorten this process, these cycles needed to occur concurrently, which have led to issues of reserving inappropriate SMEs or having insufficient time to prepare data elicitation techniques.

\section{Lesson 5: Aim for the moon vs always undersell?}

When opening lines of communication with a sponsor at the beginning of a study, there are two competing paradigms that may be followed: promising everything the sponsor wants and hoping you can achieve it; or only conceding to meet a fraction of their needs with the expectation that this will be exceeded by some amount.

In the author's opinion, LCA have a long history of applying the former. This has had a few negative effects, but also a substantial number of positive results. For the study discussed above, it perhaps would have been prudent to decline undertaking it in the first instance or to severely limit its scope.

Is one paradigm more effective than the other? Or are there definable situations in which one should be used over the other? On reflection, aiming for the moon was detrimental for the outcomes of this study. The ambitious scope put too much pressure on a minimal analytical effort and left the sponsor receiving far less in the way of analytical rigour and recommendations than they expected.

\section{CONCLUSION}

The LCA-lead SRO study of 2016 was successful in achieving some of the sponsor's requirements and unsuccessful in achieving others. The causes of failure points identified are not exceptional or novel, but they serve as a warning about how to manage a myriad of difficult situations. It is the hope that the reflections of this study may provide input into the planning process of other analysts leading and undertaking similar studies in the future. 
Williams, A critical assessment of a partially-successful analytical campaign

\section{REFERENCES}

Davies, A., Jennings, P. and Schreer, B. (2014). A versatile force: The future of Australia's special operations capability. Australian Strategic Policy Institute.

Curtis, N.J., Dortmans, P.J. and Cuik, J. (2005). 'Doing the right problem' versus 'doing the problem right': problem structuring within a Land Force environment. Journal of the Operational Research Society, 57 (11), $1300-1312$.

Department of Defence (2015). Defence White Paper 2015. http://www.defence.gov.au/WhitePaper/

Dörner, D., Nixon, P. and Rosen, D. (1990), The Logic of Failure, Philosophical Transactions of The Royal Society of London. Series B, Biological Sciences, Vol. 327, No. 1241.

Guion, L.A., Diehl, D. C. and McDonald, D. (2011), Triangulation: Establishing the Validity of Qualitative Studies, FCS6014, Institute of Food and Agricultural Sciences, University of Florida.

Kaneko, K. and Tsuda, I. (2011). Complex Systems Chaos and Beyond: A Constructive Approach with Applications in Life Sciences. Springer Science \& Business Media

Shine, D.R. and Kempt, N.K. (2017), Army Coastal, Littoral, Estuarine and Riverine (CLEAR) Capability Analysis, Rist Prize Winning presentation, Military Operations Research Society.

The Technical Cooperation Program (2006). Guide for Understanding and Implementing Defense Experimentation (GUIDeX).

Zenko, M. (2015), Red Team: How to succeed by thinking like the enemy, Basic Books. 